\title{
Las cuentas ambientales del INE: visión general, logros y retos futuros
}

\section{The Environmental Accounts of the Spanish Statistical Office: General Overview, Achievements Made and Future Challenges}

\author{
Antonio Martínez Serrano \\ Ana Luisa Solera Carnicero \\ Instituto Nacional de Estadística
}

\section{Resumen}

El Instituto Nacional de Estadística (INE) de España ha realizado un importante esfuerzo en los últimos años para desarrollar un sistema integrado de cuentas ambientales en línea con lo dispuesto en el Reglamento (UE) 691/2011 del Parlamento Europeo y del Consejo. En la actualidad se difunden anualmente siete cuentas ambientales en los ámbitos de las emisiones a la atmósfera, impuestos ambientales, flujos de materiales, gasto en protección ambiental, energía, bienes y servicios ambientales y residuos. El artículo pretende ofrecer al lector una visión global del origen de las cuentas ambientales, el marco contable donde se enmarcan los trabajos desarrollados en la Unión Europea y España en los últimos años y los importantes retos futuros que se deben afrontar.

Palabras clave: medioambiente, contabilidad ambiental, estadísticas de síntesis, cuentas satélites, flujos físicos, origen-destino, ajustes de residencia.

Clasificación JEL: JQ56.

\begin{abstract}
The National Statistical Institute of Spain has made an important effort in recent years to develop an integrated system of environmental accounts in line with the Regulation (EU) 691/2011 of the European Parliament and of the Council. Currently, seven environmental accounts are elaborated on an annual basis in the areas of emissions into the atmosphere, environmental taxes, material flows, expenditure on environmental protection, energy, environmental goods and services and waste. The article aims to provide the reader with a global view of the origin of environmental accounts, the accounting framework where they are developed, the work carried out in the European Union and Spain in recent years and the important future challenges to be faced.
\end{abstract}

Keywords: environment, environmental account, synthesis statistics, satellite accounts, physical flows, supply-use, resident adjustments. 


\section{Introducción}

El desarrollo de las sociedades ha ido unido a un incremento de la preocupación por el cuidado del entorno en el que vivimos. La necesidad de tomar decisiones adecuadas en ámbitos tan relevantes como la reducción de la contaminación, la gestión y tratamiento de los residuos generados, la disponibilidad y el uso de recursos naturales, las inversiones que realizan las empresas para conseguir procesos productivos menos agresivos con el medioambiente o el tipo de producto o actividad que va a sufrir mayor presión fiscal por el hecho de ser más contaminante, ha ocasionado en los últimos años un crecimiento exponencial de la demanda de más y mejores estadísticas relacionadas con el medioambiente.

De hecho, la mayoría de los organismos internacionales han destinado importantes recursos a la elaboración de sistemas de cuentas ambientales y económicas integrados.

La «Contabilidad Ambiental» es una operación estadística de síntesis, cuyo objetivo general es la integración de la información medioambiental de manera coherente con el Sistema de Cuentas Nacionales, siguiendo la metodología del Sistema de Contabilidad Económica y Ambiental, Marco Central (SCAE-MC) desarrollado por Naciones Unidas.

El conjunto de cuentas medioambientales permite analizar la contribución del medioambiente a la economía, así como el impacto de la economía en el medioambiente, constituyendo una herramienta para la planificación estratégica y el análisis sobre el desarrollo sostenible.

El Instituto Nacional de Estadística (INE) no ha sido ajeno a este importante incremento de la demanda de estadísticas y cuentas ambientales. Actualmente, el INE dispone de estadísticas sobre generación y tratamiento de residuos, agua y gasto en protección ambiental. Adicionalmente, elabora cuentas ambientales sobre emisiones a la atmósfera, impuestos, flujos de materiales, gasto en protección ambiental, energía, residuos y bienes y servicios medioambientales.

En el artículo se expondrá el origen del sistema de cuentas ambientales, las principales características del sistema de contabilidad ambiental y económica, la situación de las cuentas ambientales en la Unión Europea y en España para finalizar exponiendo los principales logros alcanzados en los últimos años y los retos futuros a los que nos enfrentaremos.

\section{Origen del sistema de cuentas ambientales}

A finales de los años ochenta del pasado siglo, se percibió que la contabilidad económica tradicional y los indicadores basados en ella no eran suficientes para medir el impacto que la economía y el ser humano tienen sobre el medioambiente y viceversa. Los diferentes agentes sociales y económicos empiezan a cuestionarse 
si nuestro patrón de producción y consumo era sostenible para las generaciones futuras.

Así, en el año 1987, el informe de la Comisión Brundtland titulado «Nuestro futuro común» (Comisión Mundial sobre el Medio Ambiente y Desarrollo, Brundtland, 1987), puso de manifiesto estas preocupaciones sobre el desarrollo sostenible de forma explícita.

En junio de 1992, la Conferencia de Naciones Unidas sobre el Medio Ambiente y el Desarrollo realizada en Río de Janeiro, se hace eco de esta preocupación e introduce la «Agenda 21» (Naciones Unidas, 1993), en el que se sientan las bases de lo que serían todos los trabajos posteriores de implementación de las cuentas ambientales y económicas.

En 1993, la División de Estadística de Naciones Unidas de la ONU publica con carácter provisional el Manual de Contabilidad Nacional, Ambiental y Económica Integrada (Naciones Unidas, 1993), más conocido como SCAEI.

En 1994, se crea el Grupo de Londres sobre Contabilidad Ambiental, a modo de foro de debate en donde los expertos compartan sus experiencias en el desarrollo e implementación de las cuentas ambientales, en aras de la convergencia de métodos y conceptos contenidos en el SCAEI.

En 2000, la División de Estadística de la ONU y el Programa para el Medio Ambiente de la misma entidad publican Contabilidad ambiental y económica integrada: Manual de operaciones (Naciones Unidas, 2000), con base en el material preparado por el Grupo de Nairobi (grupo de expertos establecido en 1995 integrado por agencias nacionales e internacionales y organizaciones no gubernamentales). Esta publicación reflejó la discusión permanente que se dio a raíz de la publicación del SCAEI en 1993, proponiendo formas de aplicar la contabilidad ambiental y económica integrada en la formulación de políticas. En paralelo a este trabajo, organismos internacionales, en cooperación con el Grupo de Londres, trabajaron en la revisión del SCAE versión 1993. El proceso se llevó a cabo a través de una serie de encuentros de expertos y bajo un amplio proceso de consulta.

En 2003, fruto de este trabajo conjunto fue la publicación del Manual de Contabilidad Nacional: contabilidad ambiental y económica integrada 2003 (SCAI-2003), de forma conjunta por Naciones Unidas, la Comisión Europea, el Fondo Monetario Internacional, la Organización para la Cooperación y Desarrollo Económico y el Banco Mundial (Naciones Unidas et al., 2003). Sin embargo, el hecho de que el SCAEI-2003 presentara diversas opciones metodológicas hizo que nunca se aceptara formalmente como un estándar estadístico internacional. No obstante, proporcionó un marco aceptable para la compilación de cuentas ambientales económicas que fue utilizado por muchos países en el mundo.

En 2005, en respuesta a la solicitud de los países de elevar el perfil de las cuentas ambientales, la ONU crea el Comité de Expertos de las Naciones Unidas sobre Contabilidad Ambiental y Económica (UNCEEA, por sus siglas en inglés). Se inicia así una nueva revisión del SCAEI, centrada en aquellas áreas en las que el sistema necesitaba reforzarse, tales como el registro de los flujos físicos de los recursos na- 
turales y el gasto en protección ambiental, así como el de la integración de la valoración de los servicios de los ecosistemas en la contabilidad nacional

En 2007, reconociendo que cada vez es más importante la información sobre el medioambiente y la necesidad de poner esta información en un contexto económico comprensible, para quienes toman las decisiones centrales sobre políticas, la Comisión Estadística, acordó en su 38..$^{\circ}$ periodo de sesiones, comenzar un segundo proceso de revisión con el objeto de adoptar el SCAEI como un estándar internacional para cuentas ambientales económicas dentro de los cinco años siguientes. En este proceso participaron el Grupo de Londres y el Grupo de Oslo.

En 2012, como resultado de los trabajos llevados a cabo, la Comisión de Estadística de la ONU presentó el Marco Central del SCAE (SEEA Central Framework, en inglés) (Naciones Unidas et al., 2012), el cual fue adoptado como un estándar internacional de estadística.

\section{3. ¿Qué es el marco central del sistema de contabilidad ambiental y económica?}

El marco central del sistema de contabilidad ambiental y económica (SCAE-MC), es un marco conceptual de objetivos múltiples que intenta describir las interacciones entre la economía y el medioambiente, así como el stock de activos ambientales y sus variaciones.

El SCAE-MC, mediante su estructura y utilizando un amplio conjunto de informaciones, permite la comparación y el cotejo entre los datos de origen y el desarrollo de agregados, indicadores y tendencias en un amplio conjunto de cuestiones ambientales y económicas.

El SCAE-MC intenta ofrecer de una forma sistemática y organizada toda la información ambiental y económica existente. Para ello utiliza los conceptos contables, estructuras, reglas y principios del Sistema de Cuentas Nacionales (SCN), ampliando los ámbitos específicos no recogidos por el sistema de medición contable tradicional, aportando un mayor grado de detalle, bien ofreciendo información adicional o reorganizando la ya existente, guardando la coherencia con el mismo. En ese sentido se puede llegar a hablar de un sistema satélite y a su vez totalmente coherente con los sistemas de cuentas nacionales.

Al mismo tiempo, el SCAE-MC representa una fusión de múltiples disciplinas (como la economía, la estadística, la energía, la hidrología, las ciencias ambientales, entre otras), cada una con sus propios conceptos y estructuras. Por tanto, aunque la estructura básica es la misma que la usada en el SCN, el SCAE-MC procura integrar las perspectivas de otras disciplinas para ofrecer un mejor sistema de información para el análisis económico y ambiental. Por este motivo, aparecen diferencias entre ellos que principalmente afectan al registro de los flujos físicos y monetarios y a la valoración del stock de activos ambientales y sus variaciones. 
Algunos ejemplos de estas diferencias metodológicas pueden ayudar a comprender el carácter de satélite que se le atribuye a este sistema.

Así, en el SCN solo se registran los flujos internos de las empresas, tales como la producción de bienes cuando son para uso final propio, mientras que en el SCAE se podrán registran todos los flujos internos de las empresas.

En el SCN solo se registran los bienes importados o exportados cuando existe cambio de titularidad en la propiedad de los bienes; en el SCAE siempre se han de registrar los flujos entre distintas unidades residentes, aunque no exista transferencia de la propiedad del bien importado/exportado.

En relación al stock y flujos de activos en términos monetarios, el SCAE al igual que el SCN solo incluye aquellos activos que tienen valor económico. En cambio, en términos físicos la frontera del SCAE es más amplia, ya que se incluye la tierra y todos los recursos naturales de un territorio económico que puedan suministrar recursos y espacio para las actividades económicas, siendo su valoración también más compleja que en el SCN.

Una de las características que definen al SCAE es la fuerte presencia de datos físicos, junto con los datos monetarios y de forma coherente entre sí. Sin duda ofrece ventajas, no solo por la facilidad de encontrar la información en un solo sistema, sino a la hora de realizar análisis de las interacciones entre la economía y el medioambiente. También permite calcular indicadores combinados, como por ejemplo el indicador de desacoplamiento que establece la relación entre el uso de recursos y el crecimiento del producto interior bruto de un país.

Por tanto, el SCAE es un sistema global, interdisciplinar, estructurado, pero a la vez dinámico y flexible.

Global porque los conceptos y definiciones que constituyen el SCAE-MC están elaborados de modo que puedan aplicarse en todos los países, cualquiera que sea su nivel de desarrollo económico y estadístico, su estructura económica y la composición de su medioambiente.

Interdisciplinar, dado que la integración de la información referente a la economía y al medioambiente exige un método que abarca muchas disciplinas. El SCAEMC reúne en un sistema de medición único informaciones sobre el agua, minerales, la energía, la madera, los recursos pesqueros, el suelo, la tierra y los ecosistemas, la contaminación y los desechos, la producción, el consumo y la acumulación. Para cada una de esas esferas se establecen criterios de medición detallados y específicos que están integrados en el SCAE-MC de tal forma que permitan una perspectiva general.

Estructurado, porque en la práctica, la contabilidad ambiental y económica incluye la compilación de cuadros de suministro y utilización físicos, cuentas funcionales y cuentas de activos para los recursos naturales, con una estructura definida y coherente entre sí.

Es dinámico, ya que ofrece las bases para el desarrollo de publicaciones estadísticas conexas sobre temas y asuntos específicos. El SCAE-MC se complementa con dos publicaciones: SCAE para la contabilidad experimental de los ecosistemas y SCAE Aplicaciones y Extensiones. 
Por último, es también un sistema flexible en su aplicación. El SCAE-MC está concebido como una serie de cuentas internamente consistentes e integradas, diseñadas para permitir su implementación total o por partes. Según las cuestiones ambientales concretas que se planteen, cada país puede optar por aplicar únicamente una selección de las cuentas que integran en el SCAE-MC. Como se verá más adelante, esta estrategia de aplicación flexible es por la que se ha adoptado a nivel europeo.

\section{4. ¿Qué mide el SCAE-MC?}

Como ya se ha comentado anteriormente, el Sistema de Contabilidad Ambiental y Económica, Marco Central (SCAE) describe de un modo más amplio las interacciones entre la economía y el ambiente, esto es, todos los flujos que aparecen en el Gráfico 1.

Como se observa en dicho gráfico, en el caso de los flujos físicos el Sistema permite medir los insumos naturales (flujos del ambiente a la economía), los productos (flujos producidos dentro de la economía) y los residuos (flujos de la economía hacia el ambiente). Los flujos físicos se registran utilizando el esquema de tablas de oferta y utilización, que se constituyen como tablas satélites de las tablas de origen y destino monetarias del SCN.

\section{GRÁFICO 1 \\ FLUJOS FÍSICOS DE INSUMOS NATURALES, PRODUCTOS Y RESIDUOS}

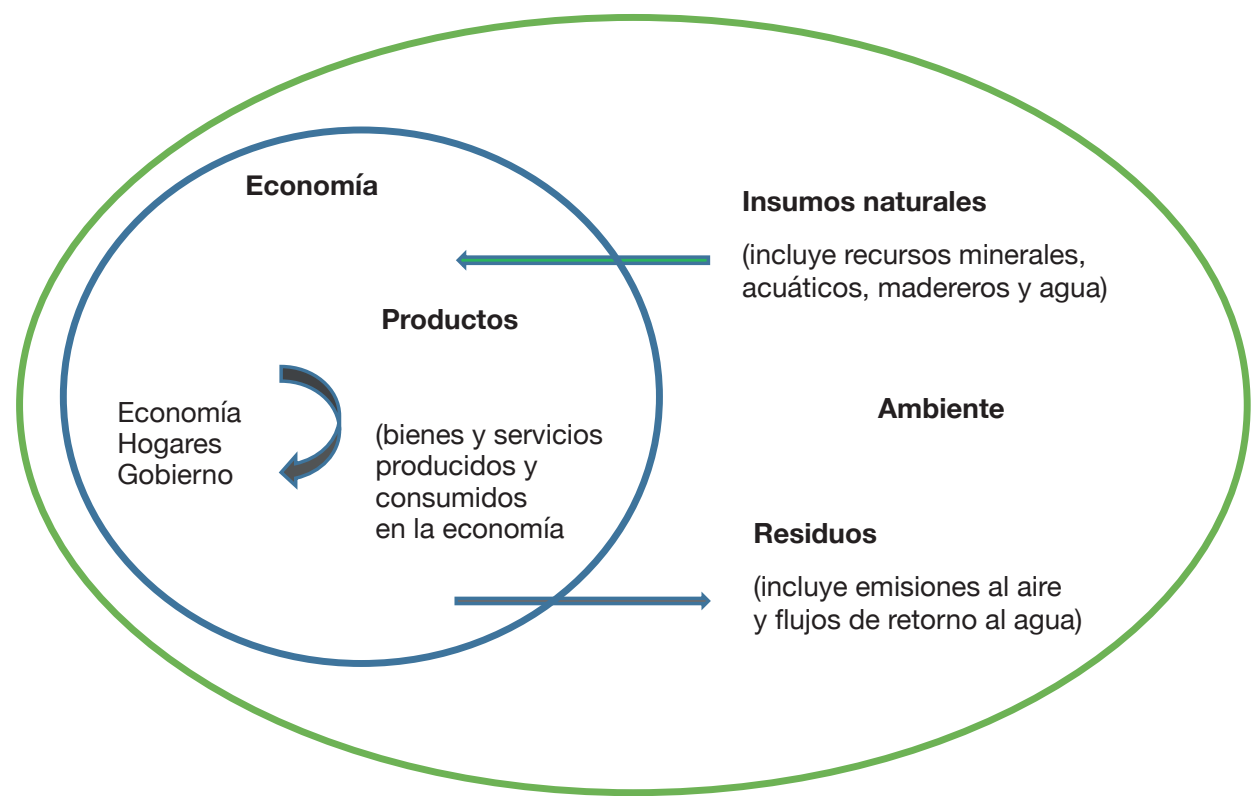

FUENTE: Naciones Unidas et al. (2016). Sistema de Contabilidad Ambiental y Económica, Marco Central. 
En relación a la actividad económica, el SCAE registra no solo el efecto que la actividad económica tiene sobre el medioambiente, sino también los flujos relacionados con las actividades económicas consideradas ambientales, tales como los gastos en protección ambiental, la producción de bienes y servicios ambientales, entre otros. Partiendo de los sistemas de cuentas tradicionales, se pueden identificar las actividades económicas realizadas con fines ambientales y presentarlas en cuentas por separado.

El uso de insumos naturales por la economía, se refleja en los cambios en el stock de activos ambientales que generan esos usos. Los activos ambientales son transformados en diferentes grados por las actividades económicas. Desde el punto de vista del SCAE se aborda la medición de los beneficios que de una forma directa e indirecta tienen los activos ambientales sobre la economía y el ambiente. Dada la complejidad de estas mediciones, se ha desarrollado el SCAE experimental de los ecosistemas que profundiza en este tipo de análisis ${ }^{1}$.

\section{Principales cuentas y cuadros del Marco Central del SCAE}

El SCAE-MC organiza e integra información sobre el stock y los diversos flujos de la economía y el ambiente en una serie de cuadros y cuentas, que se pueden agrupar en cuatro grandes grupos:

1. Cuadros de oferta y demanda (origen/destino, oferta/utilización), en unidades físicas y monetarias. Describen los flujos de insumos naturales, productos y residuos.

2. Cuentas de activos, en unidades físicas y monetarias. Muestran el stock de activos ambientales al comienzo y al final de cada periodo contable y sus variaciones.

3. Secuencia económica de cuentas, en el que destaca los agregados económicos ajustados por agotamiento. Se compilan únicamente en términos monetarios y siguen la estructura general y los mismos principios de valoración del SCN. Permiten el cálculo de saldos contables, como el valor añadido bruto, el excedente bruto de explotación, así como agregados económicos de gran relevancia como es el producto interior bruto, ajustado por agotamiento, esto es, teniendo en cuenta la disminución de las reservas de recursos naturales durante un periodo contable, debido a su extracción por parte de unidades económicas a un ritmo superior al de su regeneración. En el SCAE-MC, el valor del agotamiento se considera un coste que resta valor añadido bruto a las unidades de actividad económica extractoras de recursos naturales.

\footnotetext{
${ }^{1}$ Para una mayor información, puede consultarse la página web de Naciones Unidas relativa a la medición de los ecosistemas: https://seea.un.org/es/ecosystem-accounting
} 
4. Cuentas por función. Registran las transacciones y otras informaciones sobre actividades económicas realizadas con propósitos ambientales. Algunos ejemplos de interés son los flujos de producción de bienes y servicios ambientales, los gastos en protección ambiental y gestión de recursos, así como los impuestos y subsidios ambientales.

La información ambiental presentada por las distintas cuentas y cuadros puede verse enriquecida y ser de mayor utilidad si se relaciona con datos demográficos, sociales y de empleo. Un ejemplo puede ser la estimación del empleo generado por las actividades de producción de bienes y servicios ambientales, denominado con frecuencia «el empleo verde».

Dada la vinculación que las cuentas ambientales desarrolladas a nivel europeo, y más concretamente en nuestro país, han tenido a los cuadros de oferta y destino de los sistemas de cuentas nacionales, se describen con algo más de detalle a continuación.

\subsection{Cuadros de oferta y demanda monetarias}

Los cuadros o tablas de oferta y demanda (origen/destino) monetarias registran los flujos de productos en valores monetarios entre las diferentes unidades económicas. Se compilan para describir la estructura de la economía y el nivel de la actividad económica, si bien el SCAE-MC analiza y desagrega los más relevantes para el análisis de temas específicos ambientales.

$\mathrm{Al}$ igual que en el SCN, los flujos se «originan/ofertan» en la economía cuando:

- Son producidos por las industrias de la economía nacional (producción).

- Son introducidos desde el resto del mundo (importación).

Todos los productos ofertados tienen un uso o destino:

- Se pueden utilizar por otras industrias para elaborar productos diferentes (consumo intermedio).

- Consumir por los hogares (gasto en consumo final de los hogares).

- Consumir por las Administraciones públicas (gasto en consumo final de las Administraciones públicas).

- Vender al resto del mundo (exportaciones).

- Conservar como existencias para su uso posterior.

- Usar como activos durante un periodo de tiempo superior para producir otros productos (formación bruta de capital fijo).

Como se indica en el Cuadro 1, en las filas estos flujos se clasifican según el tipo de producto y en las columnas según el tipo de unidad económica (industrias, hogares, Administraciones públicas) y el resto del mundo. Las industrias se clasifican según su actividad principal, siguiendo el principio de residencia. 


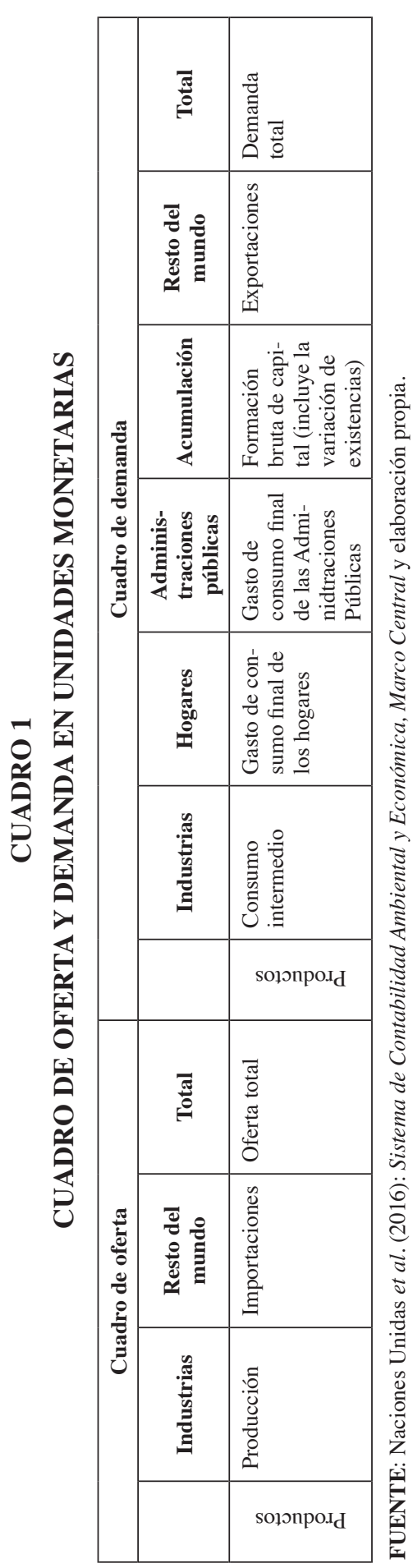


Entre las columnas consta la denominada Acumulación, que refleja los flujos ofertados en el periodo contable que no se usan durante ese periodo, sino que se acumulan en forma de existencias o de activos fijos para su uso futuro o venta a otras unidades económicas y al resto del mundo. Para una comprensión sencilla del análisis, no se han incluido las transacciones correspondientes a los impuestos y subvenciones.

En los cuadros de oferta y demanda (origen/destino) tanto monetarias como físicas se cumplen las siguientes identidades:

\section{Oferta total $=$ Demanda total}

Oferta total $=$ Producción + Importaciones

Demanda total $=$ Consumo intermedio + Gasto en consumo final de los hogares + Gasto en consumo final de las Administraciones públicas + + Formación bruta de capital + Exportaciones

A partir de las tablas se puede calcular el valor añadido bruto por industria, como la diferencia entre la producción y los consumos intermedios. La agregación de los valores añadidos nos permite obtener el producto interior bruto de la economía (PIB) desde la óptica de la oferta.

Sustituyendo en las identidades anteriores los componentes de oferta y demanda totales, obtenemos a su vez el PIB desde la óptica de la demanda:

PIB = Gasto en consumo final de los hogares + Gasto en consumo final de las Administraciones públicas + Formación bruta de capital +

+ Exportaciones - Importaciones

\subsection{Cuadros de oferta y demanda en unidades físicas}

Estos cuadros permiten evaluar la forma en la que una economía oferta y utiliza los materiales, así como examinar los cambios registrados a lo largo del tiempo en los patrones de producción y consumo. En combinación con los datos de los cuadros de oferta y demanda en unidades monetarias, se puede examinar los cambios en la productividad, entendida como la variación en el output en relación al uso de insumos naturales y de descargas de residuos.

La estructura de los cuadros de oferta y demanda en unidades físicas es similar a los compilados en unidades monetarias, si bien presentan algunas diferencias, tal y como se refleja en el Cuadro 2:

- Incorporan una columna para el ambiente y filas para los insumos naturales y las descargas de residuos.

- No figura la columna correspondiente a las Administraciones públicas, puesto que el consumo que realiza de bienes físicos está incorporado como consumo intermedio de su actividad dentro de las industrias.

- La columna de hogares también registrará la generación de residuos sólidos y otros residuos como consecuencia de su actividad de consumo. 


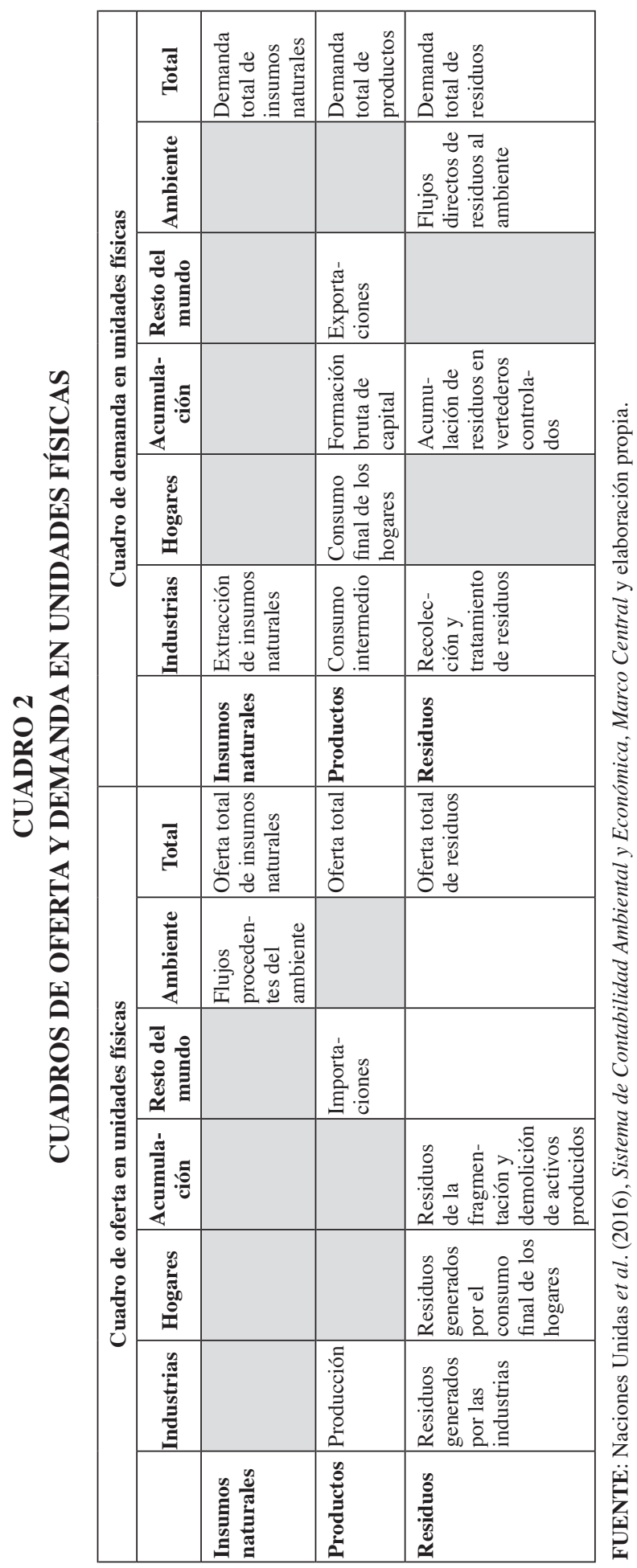


En los cuadros oferta-demanda (utilización) físicos se cumple, además de las identidades citadas en el apartado anterior para los cuadros oferta-demanda monetarios, un equilibrio adicional para los flujos entre el ambiente y la economía conocido como identidad insumo-producto. Esta identidad exige que el total de flujos hacia la economía, una empresa o un hogar, durante un periodo contable, sean acumulados en la economía o devueltos al ambiente. Se basan en la ley de conservación de la materia y la energía, que establece que en un sistema cerrado la materia y la energía permanecen constantes.

La consecuencia de esto a efectos contables consiste en que, en teoría, los flujos de materiales y de energía deben equilibrarse entre insumos naturales, productos y residuos.

Por ejemplo, los flujos de energía hacia una empresa en forma de electricidad y productos del petróleo deben liberarse en el ambiente después de ser utilizados (en forma de pérdidas por calor residual), o almacenarse (como existencias para usos futuros), o incorporarse en productos no energéticos (como los productos del petróleo utilizados para fabricar plásticos).

Los cuadros de oferta y demanda físicas se pueden centrar para analizar distintos ámbitos, como, por ejemplo, los flujos físicos de la energía o los de emisiones de gases. A diferencia de los flujos monetarios, los flujos físicos se miden en diferentes unidades según el tipo de material, lo que complica su compilación.

Las clasificaciones utilizadas para las actividades y los productos definidos en los cuadros de oferta y utilización, tanto en términos monetarios como físicos, han de ser consistentes. Así, a nivel internacional las clasificaciones utilizadas son:

- La Clasificación Industrial Uniforme de todas las actividades económicas (CIIU), para las industrias residentes en la economía nacional.

- La Clasificación Central de Productos (CPC), para los productos.

Estas clasificaciones tienen sus adaptaciones específicas tanto a nivel europeo como a nivel nacional. Así, a nivel europeo la clasificación de actividades utilizada es la Clasificación Europea de Actividades Económicas (CAE, o NACE en su versión en inglés), y para los productos se utiliza la Clasificación Europea de Productos por Actividades (CPA), que tienen su adaptación a nivel nacional a la Clasificación Nacional de Actividades Económicas (CNAE) y a la Clasificación Nacional de Productos por Actividades (CNPA) respectivamente.

No obstante, los análisis ambientales requieren la utilización de otras clasificaciones específicas, tales como la Clasificación de Actividades de Protección Ambiental (CEPA en inglés) o la Clasificación de Actividades de Gestión de los Recursos (CReMA en inglés). 


\section{Las cuentas ambientales en la Unión Europea}

Como se ha comentado al principio, la necesidad de tomar decisiones adecuadas en todos los ámbitos relacionados con la presión que el ser humano ejerce sobre el medioambiente ha hecho que la mayoría de los organismos internacionales hayan destinado importantes recursos a la elaboración de sistemas de cuentas ambientales y económicas integrados que permitan disponer de indicadores comparables.

Son muchos los ejemplos que pueden mencionarse. Así, Naciones Unidas incluyó la sostenibilidad del medioambiente dentro de los ocho principales Objetivos de Desarrollo del Milenio (Naciones Unidas, 2000). El logro de este objetivo, medido a través de distintas metas e indicadores, facilita la disponibilidad de estadísticas básicas en los distintos países.

En el caso de la Organización para la Cooperación y el Desarrollo Económico se han llevado a cabo varios foros mundiales sobre la medición del progreso de las sociedades, en los que la sostenibilidad ambiental ha sido uno de los ámbitos más relevantes.

En la Unión Europea, siete de los cuarenta y dos principales indicadores estructurales de la Estrategia de Lisboa estaban vinculados al medioambiente. El objetivo de la Estrategia de Europa 2020 (Comisión Europea, 2010) para el logro de un crecimiento inteligente y sostenible recogía, entre sus ocho principales indicadores, la reducción de un $20 \%$ de los gases de efecto invernadero, el incremento de un $20 \%$ en el uso de las energías renovables y el incremento de un $20 \%$ en la eficiencia energética. Estos objetivos han dejado paso a los recogidos en la Agenda 2030 (Naciones Unidas, 2015) para el Desarrollo Sostenible.

La Agenda plantea diecisiete objetivos con ciento sesenta y nueve metas de carácter integrado e indivisible que abarcan las esferas económica, social y ambiental. La nueva estrategia regirá los programas de desarrollo mundiales hasta 2030 y pretende, entre otros objetivos, garantizar la protección duradera del planeta y sus recursos naturales. En concreto, en el ámbito ambiental se incluyen objetivos y metas para asegurar el acceso al agua y la energía; promover el crecimiento económico sostenido y adoptar medidas urgentes contra el cambio climático.

En abril de 2008 se crea la Comisión Stiglitz-Sen-Fitoussi (Stiglitz et al., 2009) por la necesidad de ir más allá de la medición estándar del crecimiento del PIB, introduciendo alguna medida adicional que permitiera recoger el grado de progreso y bienestar alcanzado por la sociedad. Entre las recomendaciones específicas de esta Comisión figuraba la necesidad de disponer de indicadores físicos para evaluar los aspectos medioambientales de la sostenibilidad.

A raíz de estas recomendaciones, la oficina de Estadística de la Unión Europea (Eurostat) decidió crear un grupo de alto nivel para estudiar la puesta en marcha de las estadísticas y cuentas ambientales. En el marco de los trabajos que se llevaron a cabo, se consideró necesario complementar el PIB con indicadores sociales y medioambientales, así como elaborar una relación de indicadores de desarrollo sostenible, ampliando las cuentas nacionales a los ámbitos sociales y medioambientales, a través de cuentas satélites del marco central de las cuentas nacionales. 
A nivel europeo, el Sistema Europeo de Cuentas (SEC) es el principal instrumento de las estadísticas e indicadores económicos de la Unión Europea. El SEC, que es coherente con el Sistema de Cuentas Nacionales (SCN) adoptado por la División Estadística de las Naciones Unidas, es el mejor marco para analizar y evaluar distintos aspectos de la economía; sin embargo, para analizar las interacciones entre el medioambiente y la economía, el Consejo Europeo, ya en 2006, en línea con las iniciativas que se iban desarrollando en el plano de las instituciones internacionales, solicitó a sus Estados miembros que estudiaran la forma de ampliar las cuentas nacionales elaborando cuentas medioambientales, sin perder la coherencia con sistema central.

En noviembre de 2008, el Comité del Programa Estadístico, actualmente Comité del Sistema Estadístico Europeo (Eurostat, 2008) aprobó la Estrategia Europea revisada de cuentas ambientales (ESEA 2008), estableciendo las prioridades en materia ambiental, con un desarrollo progresivo y flexible de las cuentas ambientales con el fin de que todos los países europeos dispusieran de estimaciones con suficiente calidad de las cuentas medioambientales consideradas prioritarias. Fruto de esta estrategia es el Reglamento sobre cuentas económicas europeas medioambientales.

De forma paralela, la última revisión del Sistema Europeo de Cuentas (SEC 2010), se hace eco de la importancia que está adquiriendo el medioambiente e incluye un capítulo entero (el 22) dedicado a las cuentas satélites, mencionando explícitamente las cuentas ambientales y vinculándolas al aspecto funcional de la economía, esto es, al marco input-output.

Así, las cuentas ambientales europeas se establecen en el Reglamento (UE) 691/2011 del Parlamento Europeo y del Consejo, en adelante Reglamento.

El Reglamento constituye el marco jurídico para una recopilación armonizada de datos comparables de todos los Estados miembros de la UE. El Reglamento establece un marco común para la recogida, la compilación, la transmisión y la evaluación de las cuentas económicas medioambientales como cuentas satélite del SEC, proporcionando la metodología, las normas comunes, las definiciones, las clasificaciones y las normas contables destinadas a utilizarse en la compilación de estas cuentas.

Las cuentas medioambientales europeas se articulan a través de un conjunto de cuentas satélite que permiten ampliar de forma flexible la capacidad analítica de la contabilidad nacional de manera coherente con ella y sin sobrecargar el sistema central. Además, son coherentes con el SCAE-MC 2012, que ha sido adoptado como estándar estadístico internacional por los Estados miembros de la UE.

El Reglamento está estructurado en módulos (cuentas) que se han ido incorporando de forma progresiva. De hecho, en la actualidad ya se está trabajando en la ampliación del Reglamento a otros nuevos módulos.

El Reglamento entró en vigor en el año 2011 e incluía tres cuentas ambientales, referidas a emisiones a la atmósfera, flujos físicos de materiales e impuestos ambientales. En el año 2014 se aprobó la segunda fase del mismo con la incorporación de otros tres módulos: cuenta de gasto en protección ambiental, cuenta de bienes y servicios ambientales y la cuenta de flujos físicos de la energía. 
Además de estas cuentas, los países de la Unión Europea han desarrollado otras cuentas ambientales en forma de estudios piloto, que no están sometidas a un Reglamento, pero que son de interés y que posiblemente puedan incluirse en una fase posterior en el Reglamento actual. Es el caso de las cuentas de las subvenciones ambientales, las cuentas de los bosques, las cuentas del agua, las de los residuos o las cuentas de los ecosistemas entre otras.

De forma resumida, las cuentas medioambientales o módulos incluidos en el Reglamento son los siguientes:

Las cuentas de emisiones a la atmósfera (CEA) registran las emisiones a la atmósfera de seis gases de efecto invernadero incluyendo $\mathrm{CO}_{2}$ y siete contaminantes del aire, desglosadas por industrias emisoras más hogares como consumidores finales, consistente con el principio de residencia de las cuentas nacionales ${ }^{2}$.

Las cuentas de flujos de materiales para el total de la economía (CFM-TE) muestran los inputs físicos de materiales que entran en el sistema económico nacional y los outputs a otras economías o al medio natural en unidades físicas (toneladas). Esta cuenta se utiliza para estimar, entre otras cosas, las extracciones de recursos por parte de las economías, el consumo de materiales, y al relacionarlo con indicadores tales como el PIB, la productividad de los recursos y el desacoplamiento entre el crecimiento económico y la extracción de recursos. La cuenta de flujos de materiales ofrece datos tanto de la extracción nacional, como de importaciones y exportaciones de materiales.

La cuenta de impuestos ambientales presenta la desagregación por ramas de actividad y los hogares como consumidores finales, de los impuestos ambientales cuya base imponible consiste en una unidad física (o similar) de algún material que tiene un impacto negativo, comprobado y específico, sobre el medioambiente.

Las cuentas de los flujos físicos de la energía (PEFA) recogen los flujos físicos de energía (incluidos los insumos naturales utilizados para fabricar productos energéticos y residuos energéticos) del medioambiente a la economía, dentro de la economía y de la economía al medioambiente, desglosados por actividad económica y medidos en terajulios. Presentan el origen y el destino de los recursos energéticos naturales, los productos energéticos y los residuos energéticos.

Las cuentas de gastos de protección ambiental (CGPA) presentan los gastos realizados por las unidades económicas con la finalidad de proteger el medioambiente. La CGPA recoge la producción, el consumo intermedio, las importaciones y exportaciones, la inversión (formación bruta de capital fijo) y las transferencias con un desglose por cuatro sectores y por las clasificaciones funcionales de protección ambiental (CEPA).

\footnotetext{
${ }^{2}$ Los gases contaminantes considerados son: monóxido de carbono (CO), óxidos de nitrógeno (NOx), dióxido de azufre $\left(\mathrm{SO}_{2}\right)$, amoniaco $\left(\mathrm{NH}_{3}\right)$, compuestos orgánicos volátiles distintos del metano (COVNM), partículas de diámetro menor o igual a $10 \mu \mathrm{m}$ (PM10), partículas de diámetro menor o igual a 2,5 $\mu \mathrm{m}$ (PM2.5)
} 
Las cuentas del sector de bienes y servicios ambientales (CBSA) informan sobre la producción de bienes y servicios específicamente diseñados y producidos con el propósito de proteger el medioambiente o la gestión de los recursos naturales. La CBSA cubren las siguientes características: producción, exportaciones de los productos producidos, valor añadido bruto y empleo. Las cuentas tienen un desglose por industrias y por clasificaciones funcionales de protección ambiental y gestión de los recursos (CEPA y CReMA). CBSA puede ser usada para hacer un seguimiento del crecimiento de la economía y el empleo ambiental.

Para la compilación de cada una de estas cuentas, la Oficina Estadística de la Unión Europea (Eurostat) ha elaborado manuales y guías prácticas, a través de grupos de trabajo creados a tal efecto. Cada manual contiene la metodología específica para la cuenta objeto de estudio, sin perder la coherencia con el Sistema Europeo de Cuentas (SEC) y con el SCAE-MC ${ }^{3}$.

\section{Desarrollo de las cuentas ambientales en España}

Nuestro país, siendo consciente del incremento de la demanda de estadísticas y cuentas medioambientales, ha hecho un gran esfuerzo en los últimos años para la implantación de un Sistema de Estadísticas del Medio Ambiente que sirva de base al sistema de cuentas ambientales.

El Instituto Nacional de Estadística (INE) puso en marcha un área de nueva creación, a partir de los recursos disponibles en ese momento, con el objetivo de dar respuesta a las demandas de los distintos usuarios de información ambiental, tanto nacionales como internacionales, principalmente Eurostat.

Desde el principio ha sido consciente de la necesidad de formar un equipo multidisciplinar, en el que además de expertos en estadística, matemáticas y economía, se incorporaran algunos técnicos con conocimientos en otras áreas como los residuos, emisiones, agua, etc. Esto no siempre ha sido fácil, dada la escasez de recursos a la que tradicionalmente deben enfrentarse los servicios públicos. A pesar de todo, este equipo, aunque reducido en número, consiguió construir los cimientos de las estadísticas y cuentas ambientales que actualmente se elaboran en el INE.

Conviene mencionar que para la elaboración de las cuentas ambientales el INE cuenta con la colaboración de otros ministerios. Sirva como ejemplo la cuenta de emisiones a la atmósfera, construida a partir del Inventario de emisiones que elabora el Ministerio para la Transición Ecológica y Reto Demográfico (MITERD), o la cuenta de los residuos en la que parte de la información es proporcionada también por este ministerio.

Como se ha mencionado a lo largo de este artículo, las cuentas ambientales se han configurado como estadísticas de síntesis, lo que significa que intentan utilizar al máxi-

${ }^{3}$ Todos los manuales pueden consultarse en la web de Eurostat: https://ec.europa.eu/eurostat/web/ environment/methodology 
mo toda la información disponible, tanto de la Contabilidad Nacional de España como del resto de encuestas/registros administrativos existentes, adaptándolos a los criterios específicos de las cuentas medioambientales. Se trata de no sobrecargar ni al sistema de contabilidad nacional ni a los informantes con nuevas encuestas específicas.

Desde la década de los noventa y de forma gradual, se han ido elaborando estudios piloto de las distintas cuentas ambientales, en línea con los trabajos y el interés expresado por Eurostat. Estos trabajos han permitido dar cumplimiento al Reglamento.

En el año 2013 se difundieron las tres primeras cuentas del Reglamento: Cuenta de impuestos ambientales, Cuenta de emisiones a la atmósfera y Cuenta de flujos de materiales.

En el año 2017 se difundieron dos cuentas ambientales nuevas: Cuenta de Gasto en protección ambiental (CGPA) y Cuenta de flujos físicos de la energía (CFFE).

En el año 2018 se publicó la Cuenta del sector de bienes y servicios ambientales (CBSA), para la que España tenía concedida una derogación.

Las nuevas cuentas ambientales han sido presentadas al dictamen del Consejo superior de estadística (CSE), obteniendo un informe favorable. Además, están incluidas en el Plan Estadístico Nacional (PEN) y en el Inventario de Operaciones estadísticas del INE (IOE).

En la actualidad, se elaboran y difunden anualmente las seis cuentas recogidas en el Reglamento, junto con la cuenta de los residuos para el total de la economía, no estando previsto por el momento su desglose a nivel regional.

El Gráfico 2 pretende reflejar el componente satélite y de síntesis que posee la contabilidad ambiental. En el caso específico de España, además de las cuentas recogidas en el Reglamento, y dado el interés que tienen los ámbitos del agua y los bosques, se han compilado para algunos años cuentas ambientales a modo de estudios piloto que puedan servir de base para los desarrollos metodológicos de las mismas que ya se están llevando a cabo en el seno de la UE. En el caso de las cuentas de los residuos, se elaboran anualmente desde el año 2017. A partir de la información ofrecida por estas cuentas y de las cuentas nacionales, principalmente con agregados tales como el PIB, el VAB y empleo por actividades de la economía, se pueden calcular importantes indicadores ambientales.

Toda la información contenida en las cuentas ambientales se difunde en la web del $\mathrm{INE}^{4}$, por lo que ha sido necesario un trabajo adicional para dar visibilidad a estos productos nuevos, rediseñando los apartados en los que iban a estar incluidos y haciéndolos más accesibles a los usuarios de esta información. En la web se incorporan no solo los resultados actualizados, sino el informe metodológico estandarizado y una nota de prensa con las principales novedades. En los últimos años se ha hecho un importante esfuerzo para que la información sea más amigable para los distintos usuarios, elaborando infografías sobre los distintos ámbitos investigados.

\footnotetext{
${ }^{4}$ En la siguiente dirección se puede encontrar toda la información difundida por el INE en materia ambiental: https://www.ine.es/dyngs/INEbase/es/categoria.htm?c=Estadistica_P\&cid=1254735570567
} 


\section{GRÁFICO 2 \\ LAS CUENTAS AMBIENTALES EN ESPAÑA: ESTADÍSTICAS SATÉLITE DE SÍNTESIS}

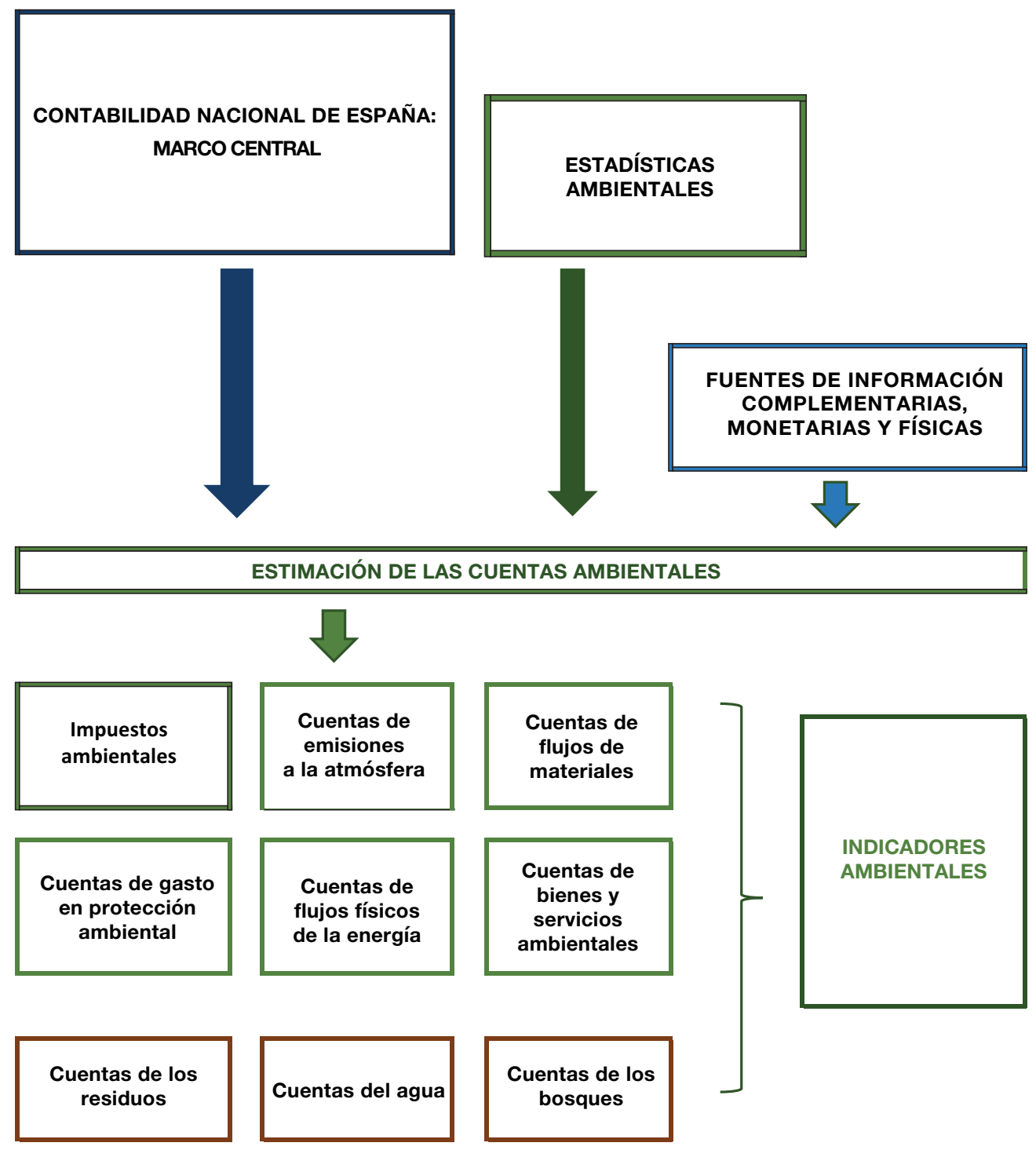

FUENTE: Elaboración propia. 
Conviene mencionar que los usuarios han valorado positivamente las importantes mejoras que se han llevado a cabo en los productos ambientales que difunde en la actualidad el INE. Según la última Encuesta de Satisfacción de Usuarios (INE, $2019)^{5}$, la valoración de los productos ambientales mejora sensiblemente respecto a encuestas anteriores.

Sin embargo, este sendero no acaba aquí, la nueva Estrategia Europea sobre cuentas ambientales ya está pensando en los desarrollos futuros del sistema de cuentas ambientales.

\section{Logros y retos futuros en el marco de la nueva Estrategia Europea sobre cuentas ambientales}

Como se ha puesto de manifiesto con anterioridad, España y los países de la Unión Europea han realizado un gran esfuerzo por implantar un nuevo sistema integrado de cuentas ambientales que ha permitido mejorar sensiblemente la cantidad y calidad de la información disponible. En concreto, en la actualidad todos los países de la UE disponen de información contable con datos comparables, completos, oportunos y transparentes en los ámbitos de emisiones a la atmósfera, impuestos ambientales, flujos de materiales, gasto en protección ambiental, energía y bienes y servicios ambientales. En los últimos años, la calidad de las seis cuentas ambientales mencionadas con anterioridad ha incrementado considerablemente debido a múltiples factores. A modo de ejemplo, merece la pena destacar los siguientes:

- La concesión de subvenciones para estudios piloto y mejoras de la calidad.

- La elaboración de estimaciones tempranas para reducir los retrasos en la disponibilidad de los datos.

- La publicación de manuales y aportación de orientación metodológica.

- El establecimiento de normas relativas a la transmisión de datos y la calidad de los informes.

- La mejora de las clasificaciones a través de grupos de trabajo específicos.

- La utilización de herramientas comunes a escala de la Unión Europea para la elaboración de resultados. Por ejemplo, la herramienta informática de uso oficial denominada PEFA-Builder, ha sido desarrollada por Eurostat para compilar las cuentas de flujos físicos de la energía sobre la base de las estadísticas sobre energía que elaboran los países de la Unión Europea siguiendo una metodología común.

- La promoción de cursos de formación en el marco del Programa Europeo de Formación Estadística.

- El fomento del intercambio de experiencias entre los distintos países.

${ }^{5}$ En el siguiente enlace se puede consultar la última Encuesta de satisfacción de los usuarios realizada por el INE: https://www.ine.es/ss/Satellite?c=Page\&cid=1259944133654\&pagename=Metodologia YEstandares\%2FINELayout\&L=0 
Además, no solo son importantes las cuentas en sí, sino los indicadores que se derivan de ellas. En base a la información contable, se pueden construir indicadores útiles para la toma de decisiones en materia de economía circular y eficiencia de los recursos. Estos indicadores permiten dar seguimiento a aspectos estratégicos a escala de la Unión Europea tan relevantes como la construcción de una economía sostenible y circular, el crecimiento verde, el cambio climático o la fiscalidad ambiental. En este sentido, podemos decir que se ha conseguido que las cuentas ambientales sean una herramienta útil para proporcionar información relevante para la toma de decisiones en aspectos vinculados al cambio climático, la contaminación del aire, energía, transporte, fiscalidad o empleo.

Sin embargo, a pesar de los logros alcanzados, existen enormes retos de cara al futuro próximo. A escala de la Unión Europea, es fundamental poder ofrecer información que permita evaluar los importantes retos que se deben enfrentar en el futuro en materia de economía circular, cambio climático o crecimiento sostenible. En concreto, en el marco del Pacto Verde (Comisión Europea, 2020), la UE aspira a elevar el nivel de ambición climática de la UE para conseguir la neutralidad climática en 2050, suministrar energía limpia, lograr una economía circular, hacer un uso eficiente de la energía y los recursos en la construcción, alcanzar una contaminación cero para un entorno sin sustancias tóxicas, preservar y restablecer los ecosistemas y la biodiversidad, garantizar un sistema alimentario sostenible y saludable y promover una movilidad sostenible e inteligente.

A escala mundial, no debemos olvidar los retos que plantea la Agenda 2030 para el desarrollo sostenible o los acuerdos existentes para la lucha contra el cambio climático. Para dar respuesta a estos retos, en febrero de 2019 el Comité del Sistema Estadístico Europeo aprobó la nueva Estrategia Europea para las cuentas ambientales (Eurostat 2019), que estará en vigor hasta $2023^{6}$. En la Estrategia se plantean importantes objetivos para el periodo 2019-2023. En concreto, se plantean los siguientes:

- Continuar mejorando la calidad de las cuentas europeas medioambientales actuales, incluidas las series cronológicas a largo plazo, la construcción de series más largas y la puntualidad.

- Comunicar mejor la pertinencia y el contenido de las cuentas europeas medioambientales, incluida la comunicación de los módulos medioambientales como un sistema completo. Para el logro de este objetivo, se considera fundamental la colaboración de la Unión Europea con otras Instituciones, el fomento del diálogo con los usuarios, la mejora en la accesibilidad de los usuarios a la información, la elaboración de indicadores teniendo en cuenta los distintos tipos de usuarios o la mejora en la interpretación y análisis de los resultados.

${ }^{6}$ En el siguiente enlace se puede consultar la Estrategia Europea para las cuentas ambientales para el periodo 2019-2023: https://ec.europa.eu/eurostat/documents/1798247/6191525/European+Strategy+for+ Environmental+Accounts/ 
- Satisfacer las necesidades de los usuarios ofreciendo más extensiones, aplicaciones e indicadores, incluidas las huellas ecológicas o el residuo alimentario.

- Evaluar la necesidad de ajustar las cuentas europeas medioambientales a las nuevas prioridades y ámbitos. En concreto, se menciona la elaboración de nuevas cuentas ambientales en otros ámbitos como los ecosistemas, las transferencias ambientales, la gestión de recursos ambientales, las cuentas de la tierra, las cuentas de los bosques o las cuentas del agua.

- Apoyar a quienes elaboran las cuentas en los Estados miembros con recursos financieros, formación, manuales y herramientas de compilación.

- Contribuir al desarrollo de las normas internacionales del Sistema de Contabilidad Económica y Ambiental y de iniciativas mundiales como el seguimiento de los Objetivos de Desarrollo Sostenible de las Naciones Unidas.

Con seguridad, de cara al futuro será necesario modificar de nuevo el Reglamento europeo para ampliar su ámbito de cobertura y poder desarrollar nuevas cuentas ambientales en la Unión Europea, y por consiguiente en España. Para ello, será necesario dotar a las Oficinas de Estadística y Ministerios competentes de recursos adecuados, mejorar la formación en la materia y continuar trabajando en los desarrollos metodológicos. En los próximos años el Instituto Nacional de Estadística continuará realizando un importante esfuerzo para afrontar los retos descritos y posicionar a España como uno de los países con mejores sistemas de cuentas ambientales de la Unión Europea y del mundo.

\section{Referencias bibliográficas}

European Statistical System Committee (2019). European Strategy for Environmental Accounts 2019-2023.

https://ec.europa.eu/eurostat/documents/1798247/6191525/European+Strategy+for+ Environmental+Accounts/

Eurostat (2002). European System for the Collection of economic information on the environment 1994 version. https://ec.europa.eu/eurostat/documents/3859598/5859717/ KS-BE-02-002-EN.PDF/468a0ed9-bdf2-4772-aa21-e04ed45c2f74?version=1.0

Eurostat (2013). European system of accounts. http://ec.europa.eu/eurostat/documents/ 3859598/5925693/KS-02-13-269- EN.PDF/44cd9d01-bc64-40e5-bd40-d17df0c69334

Eurostat, Clasificación de actividades y gastos de protección del medio ambiente (CEPA 2000). Mayo 2002.

https://www.ec.europa.eu/eurostat/ramon/nomenclatures/index.cfm?TargetUrl=LST NOM_DTL\&StrNom=CEPA_2000\&StrLanguageCode=ES\&IntPcKey $=\&$ StrLayout Code $=$ HIERARCHIC \&IntCurrentPage $=1$

Eurostat. Clasificación de Actividades Económicas (NACE Rev. 2). Recuperado de: https://www.ec.europa.eu/eurostat/ramon/nomenclatures/index.cfm?TargetUrl=LST_NOM_ DTL\&StrNom=NACE_REV2 $\&$ StrLanguageCode=ES\&IntPcKey $=\&$ StrLayoutCode $=$ HIERARCHIC\&IntCurrentPage $=1$ 
Martínez-Serrano, A., \& Solera-Carnicero, A. (2013). Las cuentas medioambientales en España: logros y retos futuros. Revista Actuarios, 33, 42-44.

Martínez-Serrano, A., Solera-Carnicero, A., \& Celestino-Rey, F. (2018). Las cuentas medioambientales y la cuenta de los flujos físicos de la energía. Economía Industrial, 408, 143-152.

Martínez-Serrano, A., \& Solera-Carnicero, A. (2019). Las cuentas medioambientales y la medición de las emisiones a la atmósfera. Revista Índice, 72, 16-19.

Naciones Unidas (1987). Informe de la Comisión Mundial sobre el Medio Ambiente y el Desarrollo «Nuestro futuro común». https://undocs.org/es/A/42/427

Naciones Unidas, Comisión Europea, Fondo Monetario Internacional, Organización para la Cooperación y Desarrollo Económico \& Banco Mundial (2009). Sistema de Cuentas Nacionales 2008. https://unstats.un.org/unsd/nationalaccount/docs/SNA2008Spanish.pdf

Naciones Unidas (2016). Sistema de Contabilidad Ambiental y Económica, Marco Central. https://www.unstats.un.org/unsd/envaccounting/seearev/CF_trans/SEEA_CF_ Final_sp.pdf

Reglamento (UE) 691/2011, del Parlamento Europeo y del Consejo, de 6 de julio de 2011, relativo a las cuentas económicas europeas medioambientales.

https://www.eur-lex.europa.eu/legal-content/ES/TXT/PDF/?uri=CELEX:32011R0691\& from $=\mathrm{EN}$

Reglamento (UE) 538/2014 del Parlamento Europeo y del Consejo, de 16 de abril de 2014, por el que se modifica el Reglamento (UE) n. ${ }^{\circ}$ 691/2011, relativo a las cuentas económicas europeas medioambientales.

https://www.eur-lex.europa.eu/legal-content/ES/ALL/?uri=CELEX:32014R0538

Tribunal de Cuentas Europeo (2019). Informe especial $n .^{\circ} 16 / 2019$. Cuentas económicas europeas medioambientales: es posible aumentar su utilidad para los legisladores. https://www.eca.europa.eu/Lists/ECADocuments/SR19_16/SR_EEEA_ES.pdf 